\title{
The Probability Distribution of Bankruptcy upon New Debt Issuances
}

\author{
Dror Parnes ${ }^{1}$ \\ ${ }^{1}$ Finance Department, College of Business, University of South Florida, USA \\ Correspondence: Finance Department, College of Business, University of South Florida, 4202 East Fowler \\ Avenue, Tampa, FL 33620, USA. Tel: 1-813-974-6357. E-mail: dparnes@usf.edu
}

Received: February 19, 2013

Accepted: March 7, $2013 \quad$ Online Published: March 18, 2013

doi:10.5539/ijef.v5n4p21

URL: http://dx.doi.org/10.5539/ijef.v5n4p21

\begin{abstract}
This study theoretically examines the probability distribution of corporate bankruptcy upon new debt issuances. We develop a relatively simple Markov model with three feasible corporate phases, derive the stochastic transition rates and the time-related probabilities to remain in each business cycle, and further simulate realistic corporate paths. We find that when both corporate debt and assets are stochastic, the probability to be in Chapter 11 is generally lower among borrowers that portray higher debt variability. Moreover, we detect that the most probable time to be in bankruptcy occurs within two or three years of a new debt issuance.
\end{abstract}

Keywords: debt issuance, bankruptcy, systematic risk, idiosyncratic risk,markov model, differential equations, simulations

\section{Introduction}

In this study we develop a rather simple theoretical model that explores the probability distribution of corporate bankruptcy upon new debt issuances. Nonetheless, our contribution does not reside within a new structural default risk model. Instead, we emphasize the universal credit consequences of corporate debt dynamics for both the borrowers and the lenders. In addition to customary derivations, we shed more light on the matter by deploying numerous computer simulations and robustness tests.

We assume this research-expedition to help corporate agents to better comprehend the potential influence of periodic changes in corporate debt on the overall likelihood to file for Chapter 11 bankruptcy protection. We associate regular oscillations in the debt level to different economic settings to direct managerial teams in their ultimate search for a higher corporate credit quality. Our notional findings further assist creditors in assessing the creditworthiness of the underlying borrowing firms. Apart from that, our theory also reveals the most likely period to be in Chapter 11 reorganization post debt issuance.

Our theory hereafter primarily predicts that whenever corporate debt is unconstrained, because of seasonal debt issuances or redemptions, and the underlying firm's assets are further stochastic, the probability to be in Chapter 11 bankruptcy protection is persistently lower among borrowers that display higher debt variability, and vice versa. Another important outcome of our model indicates that the most probable period of time to be in Chapter 11 reorganization occurs few years after a new corporate debt issuance. In most cases, the highest likelihood to be in bankruptcy protection is obtained within two or three years from debt initiation. We authenticate this notional finding for different types of debt ratios and across most practical economic circumstances. This particular observation conveys significant credit implications both for borrowers and for lenders.

This research proceeds as follows. We first propose the general theory. To gain further insight on the matter, we also deploy numerous computer simulations. Next, we conduct several robustness tests, and finally we conclude and discuss future lines of research.

\section{The Theory}

To formulate the conventional dynamics of corporate bankruptcy risk we define three distinct corporate phases with their respective probabilities per time unit to migrate from one phase to another, as follows. A firm can be either in (1) a "going concern" phase, which generally indicates normal operations, (2) a Chapter 11 reorganization phase, which designates a temporary period of bankruptcy protection yet without any liquidation at this stage, or (3) a Chapter 7 phase, which represents an absorbing state of default and a final liquidation of corporate assets. We denote the probability to file for bankruptcy protection as $\alpha$, the probability to default 
without any attempt to reorganize first as $\beta$, the probability to default while staying already in Chapter 11 as $\gamma$, and the probability to emerge from Chapter 11 back to the going concern phase as $\delta$. Since our model aims to depict a stochastic conduct of a common firm, we further assign time-related probabilities to reside in each corporate phase. We therefore denote $\varepsilon(\tau)$ as the probability to be in the going concern phase at time $\tau, \eta(\tau)$ as the probability to be in the Chapter 11 reorganization phase at time $\tau$, and $\mu(\tau)$ as the probability to be in the Chapter 7 liquidation phase at time $\tau$. For better clarity, we illustrate the feasible corporate cycles, the transition likelihoods, and the respective time-related probabilities in Figure 1.

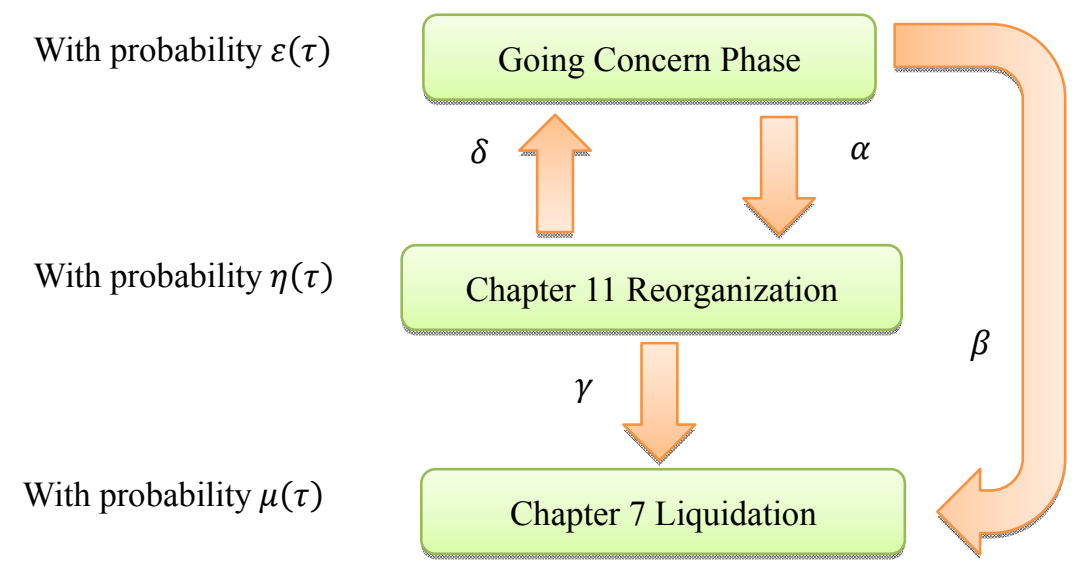

Figure 1. The model's three corporate phases and the respective migration probabilities

We can now assemble a simple Markov model of corporate survival through the following three coupled first-order differential equations:

$$
\begin{gathered}
\frac{\partial \varepsilon(\tau)}{\partial \tau}=\delta \cdot \eta(\tau)-(\alpha+\beta) \cdot \varepsilon(\tau) \\
\frac{\partial \eta(\tau)}{\partial \tau}=\alpha \cdot \varepsilon(\tau)-(\gamma+\delta) \cdot \eta(\tau) \\
\frac{\partial \mu(\tau)}{\partial \tau}=\beta \cdot \varepsilon(\tau)+\gamma \cdot \eta(\tau)
\end{gathered}
$$

Since we are interested to discover the probability distribution of corporate bankruptcy upon a new debt issuance, we shall assume that at origin the underlying firm is within the going concern phase. We therefore instigate the three initial conditions of the system as follows: $\varepsilon(\tau=0)=1, \eta(\tau=0)=0$, and $\mu(\tau=0)=0$. We are concerned about the stochastic behavior of this particular system, thus we further deploy ordinary Laplace transforms and postulate explicit derivations of the time-related probabilities as:

$$
\begin{gathered}
\varepsilon(\tau)=\left[\frac{\omega-\rho}{2 \pi} \sinh (\pi \tau)+\cosh (\pi \tau)\right] \cdot \exp \left[-\frac{(\rho+\omega) \tau}{2}\right] \\
\eta(\tau)=\frac{\alpha}{\pi} \sinh (\pi \tau) \cdot \exp \left[-\frac{(\rho+\omega) \tau}{2}\right]
\end{gathered}
$$

and from the law of total probability of mutually exclusive events we get

$$
\mu(\tau)=1-\varepsilon(\tau)-\eta(\tau)
$$

where, for simplicity, we define:

$$
\rho \stackrel{\text { def }}{=} \alpha+\beta<1
$$

as the complete exit probability from the going concern phase, which is strictly smaller than one,

$$
\omega \stackrel{\text { def }}{=} \gamma+\delta<1
$$

as the total exit probability from the Chapter 11 bankruptcy protection stage, which must be smaller than one as well due to the complement likelihood to remain within the same phase,

$$
\pi \stackrel{\text { def }}{=} \frac{\sqrt{(\omega-\rho)^{2}+4 \alpha \delta}}{2}
$$

as a temporary variable, and further recall that the hyperbolic sine and hyperbolic cosine are defined as:

$$
\sinh (\pi \tau) \stackrel{\text { def }}{=} \frac{\exp (\pi \tau)-\exp (-\pi \tau)}{2}=\frac{\exp (2 \pi \tau)-1}{2 \exp (\pi \tau)}
$$




$$
\cosh (\pi \tau) \stackrel{\text { def }}{=} \frac{\exp (\pi \tau)+\exp (-\pi \tau)}{2}=\frac{\exp (2 \pi \tau)+1}{2 \exp (\pi \tau)}
$$

At this stage of the analysis we rely on prior literature and disentangle the probability $\alpha$ to file for bankruptcy into two risk components, systematic and idiosyncratic. Jarrow and Yu (2001), Chauveau and Gatfaoui (2002), Hull and White (2004), Ou-Yang (2005), Neely and Winters (2006), Fletcher (2007), Eckner (2008), Giesecke (2008), and Parnes (2009) are among the more recent studies that scrutinize these systematic and the idiosyncratic bankruptcy risk components.

We therefore consider that firms fail to service their outstanding debt and consequently file for Chapter 11 bankruptcy protection primarily because of harsh macroeconomic conditions (systematic factors) and due to excess debt overhang and other intra-firm elements (idiosyncratic determinants). More formally we integrate the systematic and the idiosyncratic bankruptcy risk modules as:

$$
\alpha^{\text {firm }}=\alpha_{\text {sys }}^{\text {firm }}+\alpha_{i d i}^{\text {firm }}
$$

The systematic risk module of a firm naturally depends upon a specific economic state of nature, which we classify as $\widehat{\mathbb{N}}$. The corresponding idiosyncratic bankruptcy risk component logically depends upon a particular balance between the firm's latest debt level and assets following the recent bond issuance, which we categorize as $\widehat{\mathbb{D}}$. In this setting, $P(\widehat{\mathbb{D}})$ denotes the degree of debt variability, while $P(\widehat{\mathbb{D}}) \gg 0$ represents a relatively stable debt ratio and $P(\widehat{\mathbb{D}}) \ll 1$ signifies a fairly volatile debt structure. Therefore, we can now utilize the Bayes' theorem to unravel each bankruptcy risk element as the respective product of a conditional probability and a definite likelihood, as follows:

$$
\begin{aligned}
& \alpha_{\text {sys }}^{\text {firm }} \stackrel{\text { def }}{=} \alpha_{\text {sys }}^{\text {firm }} \cap \widehat{\mathbb{N}}=\left(\alpha_{\text {sys }}^{\text {firm }} \mid \widehat{\mathbb{N}}\right) \cdot P(\widehat{\mathbb{N}}) \\
& \alpha_{i d i}^{\text {firm }} \stackrel{\text { def }}{=} \alpha_{i d i}^{\text {firm }} \cap \widehat{\mathbb{D}}=\left(\alpha_{i d i}^{\text {firm }} \mid \widehat{\mathbb{D}}\right) \cdot P(\widehat{\mathbb{D}})
\end{aligned}
$$

We can further use the Merton (1974) structural credit framework, often referred as the option theoretic valuation of debt, to express the idiosyncratic conditional probability $\left(\alpha_{i d i}^{\text {firm }} \mid \widehat{\mathbb{D}}\right)$ to file for bankruptcy given a firm's specific debt ratio. In this case, we write

$$
\left(\alpha_{i d i}^{f i r m} \mid \widehat{\mathbb{D}}\right) \stackrel{\text { def }}{=} \Phi\left(-d_{2}\right)=\Phi\left[-\frac{\ln \left(\frac{A_{0}}{D}\right)+\left(r_{f}-\frac{\sigma_{A}^{2}}{2}\right) T}{\sigma_{A} \sqrt{T}}\right]
$$

where $A_{0}$ denotes the present market value of corporate assets, $D$ is the deterministic face value of debt which matures at a future time $T, r_{f}$ represents the risk-free interest rate, $\sigma_{A}$ signifies the volatility of the firm's assets, and $\Phi(\quad)$ designates the Cumulative Distribution Function (CDF) of the standard Normal distribution. In the Merton (1974) model, $\Phi\left(d_{2}\right)$ is the risk-neutral probability that the borrowing firm is capable of servicing its outstanding debt. Thus, $\Phi\left(-d_{2}\right) \equiv 1-\Phi\left(d_{2}\right)$ represents the conditional probability (given a specific debt ratio) for the underlying borrowing firm to file for bankruptcy due to intra-firm circumstances.

We can now incorporate the modified derivation for a firm's likelihood to file for Chapter 11 bankruptcy protection

$$
\alpha=\left(\alpha_{\text {sys }}^{\text {firm }} \mid \widehat{\mathbb{N}}\right) \cdot P(\widehat{\mathbb{N}})+\Phi\left[-\frac{\ln \left(\frac{A_{0}}{D}\right)+\left(r_{f}-\frac{\sigma_{A}^{2}}{2}\right) T}{\sigma_{A} \sqrt{T}}\right] \cdot P(\widehat{\mathbb{D}})
$$

into equations (4), (5), and (6) and obtain conclusive time-related probabilities to be in the going concern phase, the Chapter 11 bankruptcy protection cycle, or the Chapter 7 liquidation stage at time $\tau$ as respective functions of the likelihood to have a specific debt ratio. These relations allow us to examine the probability distribution of corporate bankruptcy under various circumstances.

A word of caution is required here though. To preserve the probability $\alpha$ to file for Chapter 11 bankruptcy within the feasible domain of $[0,1]$ we generally mandate that the idiosyncratic conditional probability of bankruptcy $\Phi\left(-d_{2}\right)$ remains sufficiently below one. In the present context, the assortment of $\Phi\left(-d_{2}\right) \rightarrow 1$ and at the same time the borrowing firm maintains a reasonably fixed debt ratio, i.e. $P(\widehat{\mathbb{D}}) \rightarrow 1$, is mathematically ill-defined because in most cases $\alpha_{\text {sys }}^{\text {firm }}>0$, which clearly violates the law of total probability. This acute setting, however, portrays a borrowing firm that cannot stay fully operational, therefore its probability distribution of bankruptcy is completely irrelevant. We therefore limit our theory to cope with those borrowing firms that have debt levels at or below market value of corporate assets. In practice, these instances are the bulk of borrowing firms, while we ought to exclude merely the extraordinary trivial cases. In our later simulations we 
demand that $D / A_{0} \leq 1$ to avoid the situation where $\Phi\left(-d_{2}\right) \rightarrow 1$, which visibly triggers $\alpha \rightarrow 1$. Regardless of other transition rates, this insignificant case essentially portrays a borrowing firm that can stay only instantaneously within the going concern corporate phase. In this particular setting the probability distribution of corporate bankruptcy is obviously extraneous.

We can expose other relations within the proposed model by realistically assuming that the probability $\beta$ to default and reach Chapter 7 liquidation directly from the going concern phase is proportional to the ad hoc debt ratio, hence $\beta \propto \frac{D}{A_{0}}$. For example, when the outstanding debt is far greater than the market value of assets, in many cases a distressed firm would abandon any attempt to reorganize and immediately liquidates its existing assets among the various classes of the debt-holders. However, we intentionally separate the emergence probability $\delta$ as well as the likelihood $\gamma$ to reach Chapter 7 and default while staying already in Chapter 11 bankruptcy protection from the firm's debt ratio. These two exit routes usually depend on the projected liquidation costs, the chances to find Debtor In Possession (DIP) financiers, and the odds to overcome various obstacles during negotiations with different classes of creditors. Alderson and Betker (1995) empirically show that firms with high liquidation costs of assets tend to emerge from Chapter 11 with relatively low debt ratios, often by raising new equity capital, while the debt of these firms is more likely to be public, unsecured, and with less restrictive covenants.

Furthermore, we notice that our derivations for the time-related probabilities to remain in each corporate cycle within equations (4), (5), and (6) are linked to the Merton (1974) structural credit model through another device. As time progresses, i.e. when $\tau$ increases, the remaining time until maturity $T$ on the firm's outstanding debt decreases. In this situation, the idiosyncratic conditional probability of bankruptcy $\Phi\left(-d_{2}\right)$ in equation (15) decreases with some convexity or concavity, depending on the precise corporate debt ratio. Consequently, the probability $\alpha$ to file for bankruptcy protection generally tends to decline. This configuration reduces the complete exit probability $\rho$ from the going concern cycle in equation (7), while these changes further affect the temporary variable $\pi$ in equation (9).

In fact, because the probability $\alpha$ to file for bankruptcy protection is a periphrastic function of the time unit $\tau$ we cannot analytically solve how $\varepsilon(\tau)$ and $\eta(\tau)$ advance through time. In various instances, however, as illustrated in the next section, we can ignore this somewhat concealed relationship between $\alpha$ and $\tau$ and differentiate by parts both time-related probabilities $\varepsilon(\tau)$ and $\eta(\tau)$. Within this venue, we can utilize the facts that $\frac{\partial}{\partial \tau} \sinh (\tau)=\cosh (\tau), \frac{\partial}{\partial \tau} \cosh (\tau)=\sinh (\tau)$, and $\frac{\partial}{\partial \tau} e^{-\tau}=-e^{-\tau}$, and acquire

$$
\begin{gathered}
\frac{\partial}{\partial \tau} \varepsilon(\tau) \cong\left[\left(\pi-\frac{\omega^{2}-\rho^{2}}{4 \pi}\right) \sinh (\pi \tau)-\rho \cosh (\pi \tau)\right] \cdot e^{-\frac{(\rho+\omega) \tau}{2}} \\
\frac{\partial}{\partial \tau} \eta(\tau) \cong\left[\cosh (\pi \tau)-\frac{\rho+\omega}{2 \pi} \sinh (\pi \tau)\right] \cdot \alpha e^{-\frac{(\rho+\omega) \tau}{2}}
\end{gathered}
$$

To find the specific point where the time-related probability $\eta(\tau)$ to be in Chapter 11 reorganization reaches a local optimum level we require that $\frac{\partial}{\partial \tau} \eta(\tau)=0$. Along this first order condition we use a simple algebra and find that an optimum time-related likelihood to stay in bankruptcy protection is achieved when

$$
\frac{\rho+\omega+2 \pi}{\rho+\omega-2 \pi}=\exp (2 \pi \tau)
$$

We notice that the ratio on the left hand side of equation (19) is independent of $\tau$ hence it is stable over time, yet the right hand side grows through time with a direct correspondence to the continuous rise in the parameter $\tau$. Thus, we can conclude that an optimum time-related probability to stay in Chapter 11 reorganization is inevitable. In addition, to obtain a local (and in this case also global) maximum we further require that $\frac{\partial^{2}}{\partial \tau^{2}} \eta(\tau)<0$. This second order condition commands that

$$
\frac{\pi}{\rho+\omega}+\frac{\rho+\omega}{4 \pi}<\frac{\cosh (\pi \tau)}{\sinh (\pi \tau)}
$$

From the definitions of hyperbolic cosine and hyperbolic sine, when $\tau$ is relatively small, the ratio on the right hand side is rather large. More formally, $\lim _{\tau \rightarrow 0} \frac{\cosh (\pi \tau)}{\sinh (\pi \tau)}=\infty$. This authorizes inequality (20) to hold and a local maximum to subsist. However, when $\tau$ is fairly large, this ratio converges quite fast to one. In the latter case, since $\lim _{\tau \rightarrow \infty} \frac{\cosh (\pi \tau)}{\sinh (\pi \tau)}=1$, a violation of inequality (20) is more probable. Altogether, we expect the highest probability to be in Chapter 11 bankruptcy protection at some early stage after the debt issuance, which ultimately depends upon the overall transition likelihoods in the system.

Nonetheless, because the probability $\alpha$ employs the CDF of the standard Normal distribution, $\alpha$ is in fact a 
non-differentiable function of $\tau$. Therefore, derivations (17) and (18) merely portray reduced-form approximations to the progressions of the time-related probabilities $\varepsilon(\tau)$ and $\eta(\tau)$ to remain within the going concern phase or the Chapter 11 reorganization cycle, respectively. In the next section we assess the merits of these approximations through a close examination of the relationship between the probability $\alpha$ to file for bankruptcy and the time measure $\tau$ for different levels of debt ratio. Yet, to better comprehend the probability distribution of corporate bankruptcy upon a new debt issuance we shall enforce the exact relations throughout the later computer simulations.

\section{Main Simulations}

We begin this section by validating the reduced-form approximations (17) and (18) for the evolvements of the time-related credit probabilities $\varepsilon(\tau)$ and $\eta(\tau)$ to remain within the going concern phase or the Chapter 11 reorganization cycle, respectively. For this purpose, we inspect through equation (16) how the probability $\alpha$ to file for bankruptcy is truly affected by the progress of the time unit $\tau$ and the consequential decline within the remaining time until debt maturity $T$, ceteris paribus. Yet, we first measure how the idiosyncratic conditional probability of bankruptcy $\Phi\left(-d_{2}\right)$ in equation (15) decreases over time with different levels of debt ratio. We therefore assign the following quantities: $A_{0}=\$ 10, D=\{\$ 2, \$ 4, \$ 6, \$ 8, \$ 10\}, r_{f}=3 \%$ per annum, $\sigma_{A}=25 \%$ per year, at origin $\tau=0$ and $T=15$ years until maturity, but when $\tau$ gradually advances to 14 years, $T$ simultaneously decreases to one year until maturity. We report the results of these simulations on the left panel of Figure 2. On the right panel of Figure 2 we further demonstrate how the complete probability $\alpha$ to file for Chapter 11 bankruptcy protection alternates with time, while in addition to the above quantities we arbitrarily select the following figures: $P(\widehat{\mathbb{N}})=0.2,\left(\alpha_{\text {sys }}^{\text {firm }} \mid \widehat{\mathbb{N}}\right)=0.4$, thus $\alpha_{\text {sys }}^{\text {firm }}=0.08$, and $P(\widehat{\mathbb{D}})=0.1$.
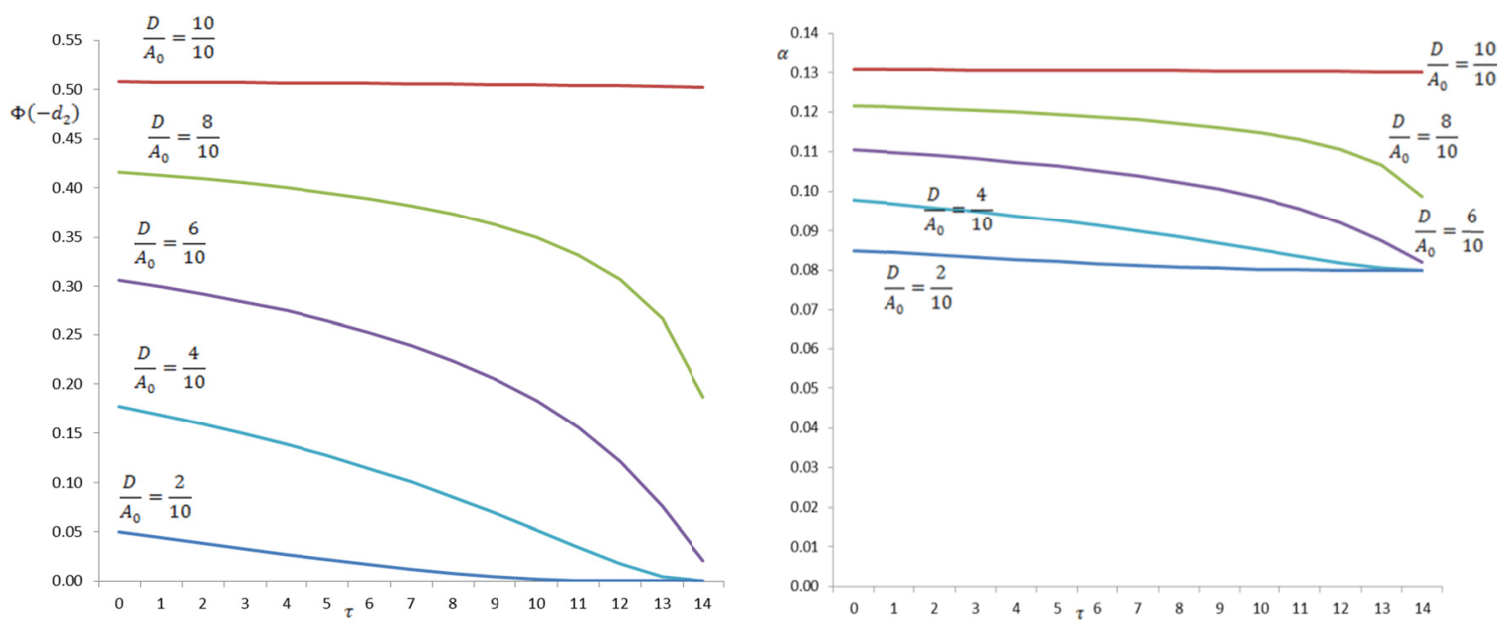

Figure 2. The evolution of bankruptcy risk over time

In the left panel we illustrate how the idiosyncratic conditional probability of bankruptcy $\Phi\left(-d_{2}\right)$ decreases over time with different levels of debt ratio. Within these simulations we arbitrarily select the following quantities: $A_{0}=\$ 10, D=\{\$ 2, \$ 4, \ldots, \$ 10\}, r_{f}=3 \%$ per annum, at origin $\tau=0$ and $T=15$ years until maturity (when $\tau$ gradually progresses to 14 years, $T$ simultaneously decreases to one year until maturity), and $\sigma_{A}=25 \%$ per annum. In the right panel we demonstrate how the probability $\alpha$ to file for bankruptcy protection moderately declines, while in addition to the above quantities we nominate the following figures: $P(\widehat{\mathbb{N}})=0.2,\left(\alpha_{\text {sys }}^{\text {firm }} \mid \widehat{\mathbb{N}}\right)=0.4$, thus $\alpha_{\text {sys }}^{\text {firm }}=0.08$, and $P(\widehat{\mathbb{D}})=0.1$. These simulations show that for relatively high or low debt ratios, the probability $\alpha$ to file for bankruptcy is reasonably autonomous of time. In these instances, equations (17) and (18) may serve as sound approximations to the evolutions of $\varepsilon(\tau)$ and $\eta(\tau)$ over time. However, for rather balanced debt ratios, the probability $\alpha$ to file for bankruptcy does vary through time. In these latter cases, equations (17) and (18) can only be used as rough estimations to the progressions of the time-related probability $\varepsilon(\tau)$ to remain in the going concern phase as well as the time-related probability $\eta(\tau)$ to be in Chapter 11 bankruptcy protection.

We can observe that when the firm's debt is relatively mobile, i.e. when $P(\widehat{\mathbb{D}}) \ll 1$, with rather high or low debt ratios, the probability $\alpha$ to file for bankruptcy is fairly independent of time. Within these occasions, equations (17) and (18) can serve as suitable approximations to the evolutions of $\varepsilon(\tau)$ and $\eta(\tau)$. However, for moderately balanced debt ratios, the probability $\alpha$ to file for bankruptcy does vary over time as a downward sloping curve. 
In these later cases, equations (17) and (18) can only be used as rough estimations for the progressions of the time-related probability $\varepsilon(\tau)$ to remain in the going concern phase as well as the time-related probability $\eta(\tau)$ to be in Chapter 11 bankruptcy protection.

Nevertheless, we learn that a fairly stable corporate debt level, i.e. when $P(\widehat{\mathbb{D}}) \rightarrow 1$, would strengthen the downward sloping curvature of the probability $\alpha$ to file for bankruptcy when measured with respect to changes in time $\tau$, since then the idiosyncratic conditional probability of bankruptcy $\Phi\left(-d_{2}\right)$ attains a bigger weight in equation (16). For these reasons, we prefer to continue our subsequent simulations with the precise derivations of corporate bankruptcy risk and not to excessively rely on these reduced-form approximations. We now turn to evaluate the notional influence of new debt issuances on corporate bankruptcy risk through equations (4) to (16).

In the next simulations we allow the borrowing firm to issue unconstrained debt while having stochastic assets. In essence, we set a fixed $\sigma_{A}>0$, and we do not restrict the debt level by disconnecting the probability $P(\widehat{\mathbb{D}})$ from all other model variables. Thus, we implicitly assume that the firm's outstanding debt can also vary over time following further debt issuances or redemptions.

We summarize the simulated results throughout Figures $3-4$ for firms having low, mid, and high debt ratios. In Figure 3 we describe how the time-related probability $\varepsilon(\tau)$ to remain in the going concern phase varies over time $\tau$ through equation (4). In Figure 4 we portray how the time-related probability $\eta(\tau)$ to be in Chapter 11 bankruptcy protection fluctuates over time $\tau$ through equation (5). For these simulations we depict hypothetical borrowing firms with relatively low, mid, and high debt ratios as: $\frac{D}{A_{0}}=\frac{\$ 2}{\$ 10}, \frac{D}{A_{0}}=\frac{\$ 5}{\$ 10}$, and $\frac{D}{A_{0}}=\frac{\$ 8}{\$ 10}$, respectively. Within each experiment we arbitrarily denote the following quantities: $r_{f}=3 \%$ per annum, $\sigma_{A}=25 \%$ per year, at origin $\tau=0$ and $T=15$ years until maturity (when $\tau$ gradually progresses to 14 years, $T$ simultaneously decreases to one year until maturity), $P(\widehat{\mathbb{D}}) \in\{0.1,0.2, \ldots, 1.0\}$ (where $P(\widehat{\mathbb{D}})=0.1$ represents a borrowing firm that exhibits a high variability of its debt level hence higher chances for further debt issuances or redemptions, and $P(\widehat{\mathbb{D}})=1.0$ characterizes a firm which preserves a constant debt ratio thus no additional debt issuances beyond the latest one), $P(\widehat{\mathbb{N}})=0.2,\left(\alpha_{\text {sys }}^{\text {firm }} \mid \widehat{\mathbb{N}}\right)=0.4$, thus $\alpha_{\text {sys }}^{\text {firm }}=0.08, \beta=\frac{D}{A_{0}} / 6=$ $\{0.0333,0.0833,0.1333\}, \gamma=0.2$, and $\delta=0.5$, thus $\omega=0.7$.

The simulated findings indicate that throughout the entire time frame under investigation and for all types of firms having unconstrained debt and stochastic assets, the time-related probability $\varepsilon(\tau)$ to remain in the going concern phase is constantly lower (higher) among firms with lower (higher) debt mobility. When the probability $P(\widehat{\mathbb{D}})$ for a specific debt level decreases (increases), hence when the debt ratio variability rises (declines), the likelihood for a borrowing firm to avoid bankruptcy and to remain fully operational increases (decreases). Furthermore, we detect that throughout the whole 14 years in our theoretical simulations and for all degrees of corporate leverage, the time-related probability $\eta(\tau)$ to be in Chapter 11 bankruptcy protection is persistently lower (higher) among borrowers having higher (lower) debt variability. These two important outcomes, however, are significantly more (less) pronounced within borrowing firms having higher (lower) debt ratios.

In addition, we detect that regardless of the specific debt ratio, the time-related probability $\varepsilon(\tau)$ to remain in the going concern cycle continuously declines as a downward sloping convex curve. This result evolves despite the fact that when time $\tau$ advances, the remaining time $T$ until the debt maturity decays, the idiosyncratic conditional likelihood $\Phi\left(-d_{2}\right)$ to file for bankruptcy decreases, and the complete probability $\alpha$ to file for bankruptcy generally drops. The continuous shrinkage in $\varepsilon(\tau)$ through time is a direct consequence of our initial condition $\varepsilon(\tau=0)=1$ and due to the absorbing state of default within the Chapter 7 phase, which gradually accumulates the chances for an irreversible liquidation over time. This corporate behavior seems highly realistic, since in practice, the vast majority of firms do fail at some point.

Even more interesting is the notional conduct of the time-related probability $\eta(\tau)$ to be in Chapter 11 reorganization. It appears that this time-related likelihood reaches its highest level shortly after initiation and it continuously decays then after. This outcome is a direct result of our initial requirement for $\eta(\tau=0)=0$ and the ergodic properties of the absorbing state of default, which essentially compel that $\varepsilon(\tau \rightarrow \infty)=0, \eta(\tau \rightarrow$ $\infty)=0$, and $\mu(\tau \rightarrow \infty)=1$. The unique curvature of $\eta(\tau)$ is obtained due to an upward sloping convex shape of the first term $\frac{\alpha}{\pi} \sinh (\pi \tau)$ and a downward sloping convex profile of the second term $\exp \left[-\frac{(\rho+\omega) \tau}{2}\right]$ within equation (5). 

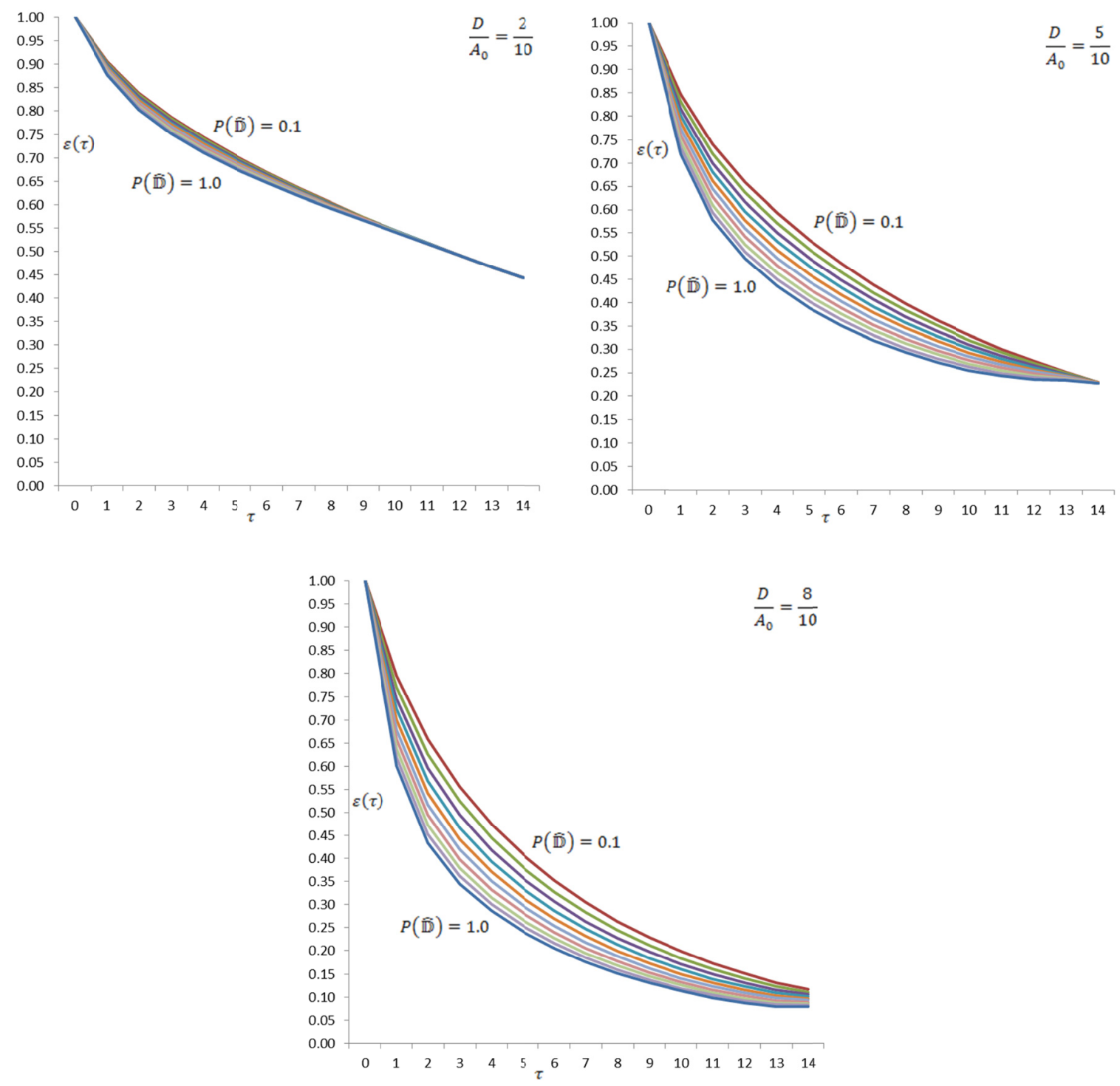

Figure 3. Time-related operational probabilities with unconstrained low, mid, and high debt ratios

In the upper left, upper right, and lower panels we describe how the time-related probability $\varepsilon(\tau)$ to remain in the going concern corporate phase changes over time $\tau$ through equation (4) with low, mid, and high debt ratios, respectively. For these simulations we depict hypothetical firms having the following arbitrary quantities: $A_{0}=\$ 10, D=\{\$ 2, \$ 5, \$ 8\}, r_{f}=3 \%$ per annum, at origin $\tau=0$ and $T=15$ years until maturity (when $\tau$ gradually progresses to 14 years, $T$ simultaneously decreases to one year until maturity), $\sigma_{A}=25 \%$ per year, $P(\widehat{\mathbb{D}}) \in\{0.1,0.2, \ldots, 1.0\}$ (where $P(\widehat{\mathbb{D}})=0.1$ represents a borrowing firm that exhibits a high variability of its debt hence further debt issuances or redemptions are likely, and $P(\widehat{\mathbb{D}})=1.0$ characterizes a borrowing firm which preserves a fixed debt level), $P(\widehat{\mathbb{N}})=0.2,\left(\alpha_{\text {sys }}^{\text {firm }} \mid \widehat{\mathbb{N}}\right)=0.4$, thus $\alpha_{\text {sys }}^{\text {firm }}=0.08, \beta=\frac{D}{A_{0}} / 6=$ $\{0.0333,0.0833,0.1333\}, \gamma=0.2$, and $\delta=0.5$, thus $\omega=0.7$. These experiments reveal how the time-related probability $\varepsilon(\tau)$ to remain in the going concern phase decay over time, while higher debt variability is essential for staying fully operational, especially among the high debt ratio firms. 

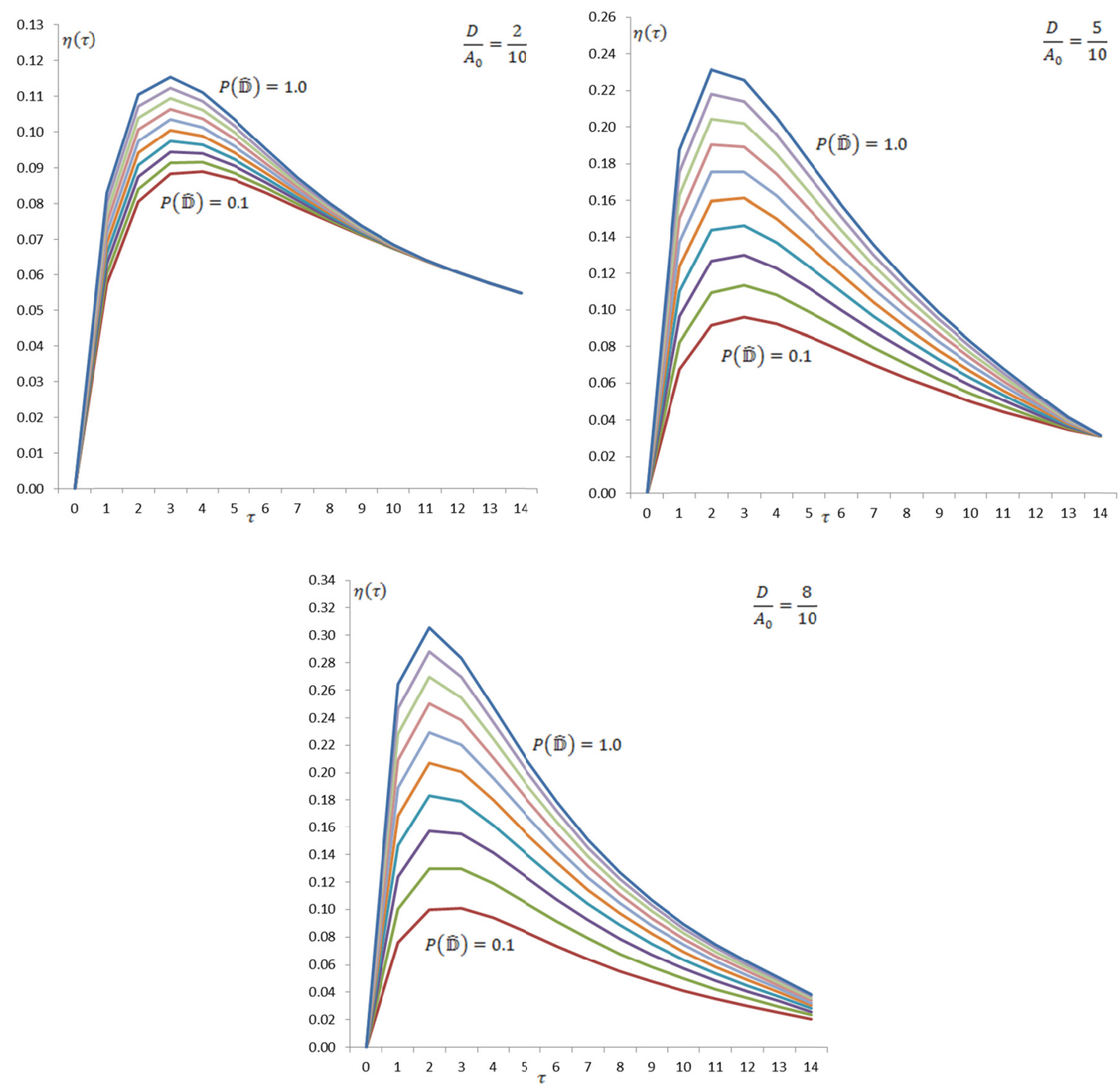

Figure 4. Time-related bankruptcy probabilities with unconstrained low, mid, and high debt ratios

In the upper left, upper right, and lower panels we describe how the time-related probability $\eta(\tau)$ to be in Chapter 11 bankruptcy protection changes over time $\tau$ through equation (5) with low, mid, and high debt ratios, respectively. For these simulations we depict hypothetical firms having the following arbitrary quantities: $A_{0}=\$ 10, D=\{\$ 2, \$ 5, \$ 8\}, r_{f}=3 \%$ per annum, at origin $\tau=0$ and $T=15$ years until maturity (when $\tau$ gradually progresses to 14 years, $T$ simultaneously decreases to one year until maturity), $\sigma_{A}=25 \%$ per year, $P(\widehat{\mathbb{D}}) \in\{0.1,0.2, \ldots, 1.0\}$ (where $P(\widehat{\mathbb{D}})=0.1$ represents a borrowing firm that exhibits a high variability of its debt hence further debt issuances or redemptions are likely, and $P(\widehat{\mathbb{D}})=1.0$ characterizes a borrowing firm which preserves a fixed debt level), $P(\widehat{\mathbb{N}})=0.2,\left(\alpha_{\text {sys }}^{\text {firm }} \mid \widehat{\mathbb{N}}\right)=0.4$, thus $\alpha_{\text {sys }}^{\text {firm }}=0.08, \beta=\frac{D}{A_{0}} / 6=$ $\{0.0333,0.0833,0.1333\}, \gamma=0.2$, and $\delta=0.5$, thus $\omega=0.7$. These experiments authenticate how the time-related probabilities $\eta(\tau)$ to be in Chapter 11 bankruptcy protection first increase and then decrease over time, while higher debt variability is essential for avoiding Chapter 11 bankruptcy protection, especially among the high debt ratio firms.

Furthermore, we notice that the maximum time-related probability $\eta(\tau)$ to be in Chapter 11 bankruptcy protection is repeatedly achieved within two or three years of a new debt issuance. As predicted by the theory in derivations (19) and (20), this result remains robust regardless of the specific debt ratio or the firm's debt variability. We provide some pragmatic intuition to this phenomenon by realizing that in most cases, borrowing firms can issue new debt only when they do not convey an immediate bankruptcy risk. These leveraged firms, 
however, damage their own creditworthiness with every debt issuance, thus their credit quality deteriorates shortly after. Nevertheless, when enough time has passed since the debt issuance, the borrower has either adjusted its core business to the new debt level or defaulted and completely liquidated its assets.

\section{Robustness Tests}

Throughout the main simulations thus far we have picked arbitrary values for the model variables. In particular, we have frequently used the following pivot numbers: $\mathrm{r}_{\mathrm{f}}=3 \%$ per annum, $\sigma_{\mathrm{A}}=25 \%$ per year, $\gamma=0.2$, $\delta=0.5,\left(\alpha_{\text {sys }}^{\text {firm }} \mid \widehat{\mathbb{N}}\right)=0.4, \mathrm{P}(\widehat{\mathbb{N}})=0.2, \mathrm{~T}=15$ years until maturity, and $\beta=\frac{\mathrm{D}}{\mathrm{A}_{0}} / 6$. These subjectively selected measures depict reasonable quantities that aim to represent common observed patterns.

Nonetheless, for purpose of robustness, we wish to test our theory with alternative feasible values. We therefore alternate each variable through a large spectrum of practical measures. More formally, we reproduce the previous simulations with the following sets of quantities: $r_{f} \in\{1 \%, 2 \%, \ldots, 15 \%\}$ per annum, $\sigma_{\mathrm{A}} \in\{5 \%, 10 \%, \ldots, 40 \%\} \quad$ per $\quad$ year, $\gamma \in\{0.05,0.10, \ldots, 0.50\}, \quad \delta \in\{0.05,0.10, \ldots, 0.50\}, \quad\left(\alpha_{\text {sys }}^{\text {firm }} \mid \widehat{\mathbb{N}}\right) \in$ $\{0.1,0.2, \ldots, 0.6\} \quad, \quad \mathrm{P}(\widehat{\mathbb{N}}) \in\{0.1,0.2, \ldots, 0.6\} \quad, \quad \mathrm{T} \in(5,10, \ldots, 25)$ years until maturity, and $\beta \in\left\{\frac{\mathrm{D}}{2 \times \mathrm{A}_{0}}, \frac{\mathrm{D}}{4 \times \mathrm{A}_{0}}, \ldots, \frac{\mathrm{D}}{20 \times \mathrm{A}_{0}}\right\}$. These robustness tests do not yield materially different outcomes than the already testified results from the main simulations.

There are, however, a few interesting points to notice here. First, when the risk free interest rate reaches exceedingly high levels, near $15 \%$ or so, the differences between low and high debt variability become negligible. With exceptionally high $r_{f}$ the conditional probability $\Phi\left(-d_{2}\right)$ to file for bankruptcy converges to zero in equation (15). In this case, the integrated likelihood $\alpha$ to file for Chapter 11 bankruptcy protection stabilizes in equation (16), while the effects of different debt dynamics on corporate bankruptcy risk are virtually abolished.

Second, when the two exit routes from Chapter 11 are reduced to extraordinarily low levels, i.e. when the probabilities $\gamma$ or $\delta$ fall below 0.05 or so, the time-related probability $\eta(\tau)$ to be in Chapter 11 reorganization attains its maximum level somewhat later than before, around four to five years after initiation. The reason for this phenomenon lies in the fact that with these lowered transition rates $\gamma$ and $\delta$, any borrowing firm that enters the bankruptcy phase is evidently assumed to remain in this corporate cycle for a longer period of time. In this setting, the time-related probability $\eta(\tau)$ to be in Chapter 11 bankruptcy protection reaches its highest level a bit later than throughout the main simulations. Nonetheless, this maximum likelihood is still achieved within the first few years, as anticipated by the theory.

\section{Summary}

In this study we have theoretically examined the probability distribution of corporate bankruptcy upon new debt issuances. For this purpose, we have developed a relatively simple Markov model with three feasible corporate phases: (1) a going concern cycle, (2) a Chapter 11 bankruptcy protection stage, and (3) an absorbing state of default within Chapter 7 liquidation. We have presented analytical solutions for the stochastic transition rates among these corporate phases and linked the continuous dynamics of corporate debt to time-related probabilities to remain in each cycle. In addition, we have offered several reduced-form approximations that can crudely predict bankruptcy risk patterns among various borrowing firms. To better comprehend the behavioral properties of the system, we have further simulated the model derivations. Finally, we have authenticated our predictions and deployed numerous robustness tests by alternating the model variables through large sensible ranges.

Overall, the notional findings of our model project that when corporate debt is unconstrained due to seasonal debt issuances or redemptions and the firm's assets are further stochastic, the time-related probability to be in Chapter 11 bankruptcy protection is persistently lower among borrowers that portray higher debt variability, and vice versa.

A valuable product of our theory indicates that the most probable period of time to file for Chapter 11 reorganization develops a small number of years after a new corporate debt issuance. In most cases, the highest likelihood to be in bankruptcy protection is realized within two or three years of debt initiation. We ascertain this vigorous result for all types of debt ratios and across most practical economic circumstances. This perception conveys significant credit implications for both borrowers and lenders. Both of these counterparties should brace themselves individually for this hazardous corporate phase and further direct proactive measures to mitigate certain credit implications.

As future lines of research we recommend that interested parties pursue empirical tests of the prophecies of the current theory. This however, may not be a trivial journey. While debt variability over time can be measured with 
relatively naïve statistical techniques, the assessment of its impact on corporate bankruptcy risk might burden quite a few difficulties. Despite a universal agreement throughout the economic literature that corporate bankruptcy risk evolves both from systematic factors and idiosyncratic determinants, it may not be a straightforward task to isolating the marginal contributions in practice. Since these two risk modules are not readily observed, it could be somewhat challenging to empirically attribute the isolated influence of debt variability on the overall risk to file for Chapter 11 bankruptcy protection. For that reason, our study remains a theoretical exercise.

\section{References}

Alderson, M. J., \& Betker, B. L. (1995). Liquidation Costs and Capital Structure. Journal of Financial Economics, 39, 45-69. http://dx.doi.org/10.1016/0304-405X(94)00825-L

Chauveau, T., \& Gatfaoui, H. (2002). Systematic Risk and Idiosyncratic Risk: A Useful Distinction for Valuing European Options. Journal of Multinational Financial Management, 12, 305-321. http://dx.doi.org/10.1016/S1042-444X(02)00013-0

Eckner, A. (2008). Risk Premia in Structured Credit Derivatives. Working paper, Stanford University.

Fletcher, J. (2007). Can Asset Pricing Models Price Idiosyncratic Risk in U.K. Stock Returns? Financial Review, 42, 507-535. http://dx.doi.org/10.1111/j.1540-6288.2007.00181.x

Giesecke, K. (2008). Portfolio Credit Risk: Top Down vs. Bottom Up Approaches. In Wiley R. C. (Ed.), Frontiers in Quantitative Finance: Credit Risk and Volatility Modeling.

Hull, C. J., \& White, A. D. (2004). Valuation of a CDO and an n-th to Default CDS without Monte Carlo Simulation. Journal of Derivatives, 12, 8-23. http://dx.doi.org/10.3905/jod.2004.450964

Jarrow, R. A., \& Yu, F. (2001). Counterparty Risk and the Pricing of Defaultable Securities. The Journal of Finance, 56, 1765-1799. http://dx.doi.org/10.1111/0022-1082.00389

Merton, R. C. (1974). On the Pricing of Corporate Debt: The risk Structure of Interest Rates. The Journal of Finance, 29, 449-470.

Neely, C. J., \& Winters, D. B. (2006). Year-End Seasonality in One-Month LIBOR Derivatives. Journal of Derivatives, 13, 47-65. http://dx.doi.org/10.3905/jod.2006.616867

Ou-Yang, H. (2005). An Equilibrium Model of Asset Pricing and Moral Hazard. Review of Financial Studies, 18 , 1253-1303.

Parnes, D. (2009). The Systematic and Idiosyncratic Modules of Bankruptcy Risk. Journal of Credit Risk, 5, $25-46$. 